\title{
Perfusion in hand arthritis on dynamic contrast-enhanced computed tomography: a randomized prospective study using MRI as a standard of reference
}

\author{
Sevtap Tugce Ulas ${ }^{1} \cdot$ Kay Geert Hermann ${ }^{1} \cdot$ Marcus R. Makowski ${ }^{1} \cdot$ Robert Biesen $^{2} \cdot$ Fabian Proft $^{3} \cdot$ Ralph Schilling $^{4}$. \\ Torsten Diekhoff ${ }^{1}$ (i)
}

Received: 1 April 2020 /Revised: 15 June 2020 / Accepted: 18 June 2020 / Published online: 30 June 2020

(C) The Author(s) 2020

\begin{abstract}
Objective To evaluate the performance of dynamic contrast-enhanced CT (DCE-CT) in detecting and quantitatively assessing perfusion parameters in patients with arthritis of the hand compared with dynamic contrast-enhanced MRI (DCE-MRI) as a standard of reference.

Materials and methods In this IRB-approved randomized prospective single-centre study, 36 consecutive patients with suspected rheumatoid arthritis underwent DCE-CT (320-row, tube voltage $80 \mathrm{kVp}$, tube current $8.25 \mathrm{mAs}$ ) and DCE-MRI $(1.5 \mathrm{~T})$ of the hand. Perfusion maps were calculated separately for mean transit time (MTT), time to peak (TTP), relative blood volume (rBV), and relative blood flow (rBF) using four different decomposition techniques. Region of interest (ROI) analysis was performed in metacarpophalangeal joints II-V and in the wrist. Pairs of perfusion parameters in DCE-CT and DCE-MRI were compared using a two-tailed $t$ test for paired samples and interpreted for effect size (Cohen's d). According to the Rheumatoid Arthritis Magnetic Resonance Imaging Score (RAMRIS) scoring results, differentiation of synovitis-positive and synovitis-negative joints with both modalities was assessed with the independent $t$ test.

Results The two modalities yielded similar perfusion parameters. Identified differences had small effects (d 0.01-0.4). DCE-CT additionally differentiates inflamed and noninflamed joints based on $\mathrm{rBF}$ and $\mathrm{rBV}$ but tends to underestimate these parameters in severe inflammation. The total dose-length product (DLP) was $48 \mathrm{mGy} * \mathrm{~cm}$ with an estimated effective dose of $0.038 \mathrm{mSv}$.

Conclusion DCE-CT is a promising imaging technique in arthritis. In patients with a contraindication to MRI or when MRI is not available, DCE-CT is a suitable alternative to detect and assess arthritis.
\end{abstract}

Keywords Computed tomography $\cdot$ Magnetic resonance imaging $\cdot$ Perfusion imaging $\cdot$ Arthritis, rheumatoid $\cdot$ Synovitis

Torsten Diekhoff

torsten.diekhoff@charite.de

1 Department of Radiology, Charité - Universitätsmedizin Berlin, Campus Mitte, Humboldt - Universität zu Berlin, Freie Universität Berlin, Berlin, Germany

2 Department of Rheumatology, Charité - Universitätsmedizin Berlin, Campus Mitte, Humboldt - Universität zu Berlin, Freie Universität Berlin, Berlin, Germany

3 Department of Rheumatology, Charité - Universitätsmedizin Berlin, Campus Benjamin Franklin, Humboldt - Universität zu Berlin, Freie Universität Berlin, Berlin, Germany

4 Institute of Biometry and Clinical Epidemiology, Charité Universitätsmedizin Berlin, Campus Mitte, Humboldt - Universität zu Berlin, Freie Universität Berlin, Berlin, Germany

\section{Introduction}

The early detection of inflammatory changes such as synovitis is crucial to prevent the development of structural changes and bone destruction such as erosions [1]. Qualitative evaluation of inflammatory activity is performed using the American College of Rheumatology/European League Against Rheumatism collaborative initiative (ACR/EULAR) criteria [2]. Dynamic contrast-enhanced imaging techniques enable the assessment of synovial enhancement over time in patients with rheumatoid arthritis [3-5]. As a result, a comprehensive evaluation of inflammatory activity in rheumatic joint diseases is possible [6-8].

With dynamic contrast-enhanced magnetic resonance imaging (DCE-MRI), it has become possible to evaluate synovitis and disease activity with sufficient accuracy [9] to 
quantify the response to therapy in research studies. Thus far, dynamic contrast-enhanced imaging has not been used widely to assess patients with arthritis in clinical practice. One reason is that sophisticated imaging technology and special software tools are required to derive and assess perfusion parameters. Ultrasonography can be offered as an alternative imaging test [10], but the results are often dependent on the experience of the examiner and difficult to quantify [11]. The first studies investigated the capability of computed tomography (CT) in the detection of active inflammation [12, 13]. Dynamic contrast-enhanced computed tomography (DCE-CT) has the potential to provide a differentiated estimation of inflammatory joint lesions through the assessment of perfusion parameters. Furthermore, it offers the superior detection of structural changes intrinsic to CT [14-16]. The use of DCE-CT in clinical practice has become feasible due to dose reduction with low-kVp scanning protocols and the introduction of iterative reconstruction algorithms in recent years [17, 18].

The purpose of our study was to evaluate the potential of lowdose DCE-CT in the detection and evaluation of synovitis of the hand in patients with acute arthritis using DCE-MRI as the standard of reference. CT offers a sensitive alternative to MRI with lower costs, wider availability, shorter examination duration, and superior detection of erosions at the cost of radiation exposure.

\section{Materials and methods}

\section{Subjects}

In our randomized prospective single-centre study, we consecutively investigated 37 patients (according to a calculation of the necessary sample size) with suspected or proven diagnosis of rheumatoid arthritis (RA) and fulfilled the classification criteria of the American College of Rheumatology (ACR) [19]. The patients presented to the rheumatology inpatient or outpatient clinic of our institution from September 2016 to October 2017. Due to the request of the local ethics committee, all patients had to be over 50 years old. Exclusion criteria were contraindications to MRI, e.g. pacemaker or cochlear implants, known allergic reactions to contrast agent as well as patients with reduced kidney function (glomerular filtration rate $(\mathrm{GFR})<60 \mathrm{ml} / \mathrm{min} / 1.73 \mathrm{~m}^{2}$ ) and hyperthyroidism. The final diagnosis was made by board-certified rheumatologists based on clinical (patient's history, number of swollen and tender joints) and laboratory findings (C-reactive protein, rheumatoid factors (RF) and anti-citrullinated protein antibodies (ACPA)) as well as imaging findings of our study.

The study was approved by the local ethics committee (EA1/259/15) and the Federal Office for Radiation Protection (Z 5-22,462/2-2016-008). All patients gave their written informed consent.

\section{DCE-CT and DCE-MRI procedures}

All patients underwent DCE-CT and 1.5 T DCE-MRI of the clinically dominant hand and wrist. Both scans were performed on the same day with a maximum time interval of $1 \mathrm{~h}$ between them. Patients were randomized to DCE-CT first or DCE-MRI first.

Patients were scanned in a prone position with the hand stretched above the head into the scanner and positioning the palm on the table (so-called superman position). The arm was fixed with a sandbag to reduce motion. Contrast agent was administered into the contralateral arm at a flow of $2 \mathrm{ml} / \mathrm{s}$ using an intravenous needle (Braunüle $\AA, 1.10 \times 33 \mathrm{~mm}$, G20, Vasofix ${ }^{\circledR}$, B. Braun ${ }^{\circledR}$; Melsungen, Germany) and an automatic injection device.

\section{Dynamic contrast-enhanced CT acquisition protocol}

DCE-CT was performed on a 320-row CT scanner (Canon Aquilion ONE Vision, Canon Medical Systems; Otawara, Japan) in volume mode with $16 \mathrm{~cm}$ z-axis coverage without table movement. We used a tube voltage of $80 \mathrm{kVp}$ and a tube current of $30 \mathrm{~mA}$ with a rotation time of $0.275 \mathrm{~s}$. A total of 10 imaging volumes were acquired. The scans were obtained $0,10,20,30$, $40,50,60,90,120$, and $180 \mathrm{~s}$ after contrast injection. We administered a body-weight-adapted dose of $1 \mathrm{ml} / \mathrm{kg}$ iopromide (Ultravist $370 \AA$, Bayer $\AA$; Leverkusen, Germany) directly followed by a bolus of $30 \mathrm{ml}$ sodium chloride. Images were reconstructed with a slice thickness of $0.5 \mathrm{~mm}$ using a medium soft tissue kernel without beam hardening compensation.

\section{Dynamic contrast-enhanced MRI acquisition protocol}

DCE-MRI was performed on a 1.5-T Siemens Magnetom Avanto imager (Siemens Healthcare; Erlangen, Germany) using a small flexible 4-channel coil (Siemens healthcare; Erlangen, Germany) for the hand.

Before contrast agent injection, we acquired the following sequences: T1-weighted spin-echo sequences in coronal planes and coronal T1-weighted turbo-inversion recoverymagnitude sequences (TIRM) (for details see Table 1).

For perfusion analysis, we acquired coronal fat-saturated dynamic T1-weighted fast 3D gradient-echo volumetric interpolated breath-hold examination (dVIBE) sequences after contrast medium injection. VIBE sequences were obtained every $10 \mathrm{~s}$ over $3 \mathrm{~min}$. Postcontrast images were acquired with fat-saturated T1-weighted spin-echo sequences in coronal and transverse slice orientations.

Contrast medium was diluted in isotonic sodium chloride solution at a ratio of 1:4, and an amount of $1 \mathrm{ml} / \mathrm{kg}$ was injected. This resulted in a total contrast agent volume of $0.2 \mathrm{ml} / \mathrm{kg}$ body weight of gadolinium-DOTA (Dotarem®; Guerbet, France) and sodium chloride volume of $0.8 \mathrm{ml} / \mathrm{kg}$ body weight. Thereafter, a bolus of $30-\mathrm{ml}$ pure sodium chloride solution was administered. 
Table 1 DCE-MRI protocol

\begin{tabular}{|c|c|c|c|c|c|c|c|c|}
\hline & $\begin{array}{l}\text { FOV Read } \\
{[\mathrm{mm}]}\end{array}$ & $\begin{array}{l}\text { FOV Phase } \\
{[\%]}\end{array}$ & $\begin{array}{l}\text { Slice thickness } \\
{[\mathrm{mm}]}\end{array}$ & $\begin{array}{l}\text { Number of } \\
\text { Slices }\end{array}$ & $\begin{array}{l}\text { TR } \\
{[\mathrm{msec}]}\end{array}$ & $\begin{array}{l}\mathrm{TE} \\
{[\mathrm{msec}]}\end{array}$ & $\begin{array}{l}\text { Resolution } \\
\text { Matrix }\end{array}$ & $\begin{array}{l}\text { Flip Angle } \\
\text { [degree] }\end{array}$ \\
\hline $\mathrm{T} 1$ & 160 & 100 & 3 & 12 & 401 & 21 & $512 \times 512$ & 90 \\
\hline TIRM & 160 & 100 & 3 & 16 & 5000 & 69 & $256 \times 241$ & 150 \\
\hline $\begin{array}{l}\text { dVIBE } \\
\text { T1 fs }\end{array}$ & 180 & 75 & 3 & 20 & 6.5 & 2.53 & $192 \times 128$ & 17 \\
\hline Coronal & 200 & 100 & 3 & 14 & 719 & 11 & $512 \times 256$ & 150 \\
\hline Axial wrist & 140 & 100 & 4 & 14 & 591 & 15 & $320 \times 192$ & 90 \\
\hline $\begin{array}{l}\text { Axial } \\
\text { MCP }\end{array}$ & 140 & 100 & 4 & 12 & 507 & 15 & $320 \times 192$ & 90 \\
\hline
\end{tabular}

FOV field of view, TR repetition time, TE echo time, TIRM turbo-inversion recovery-magnitude, $S W I$ susceptibility-weighted imaging, VIBE volumetric interpolated breath-hold examination, $T 1 \mathrm{~T} 1$-weighted imaging, $T 1 f s$ fat-saturated contrast-enhanced $\mathrm{T} 1$ sequence

\section{Image reading}

Prior to quantitative perfusion analysis, three independent readers (K.G.H. with 18 years, T.D. with 9 years, S.T.U. with 1 year of experience in musculoskeletal imaging) blinded to identifying information, clinical data, and other imaging findings scored the fat-saturated contrast-enhanced T1-weighted images for synovitis according to the Rheumatoid Arthritis Magnetic Resonance Imaging Score (RAMRIS) criteria, defined by the Outcome Measurement in Rheumatology (OMERACT) MRI group [20] to dichotomize the joints in inflamed (RAMRIS synovitis $\geq 1$ ) versus noninflamed (RAMRIS synovitis $=0$ ). The agreement of at least two readers was determined as a reference.

\section{Image preprocessing}

All images were pseudonymized with regard to name, age, and sex. Preprocessing for perfusion maps in DCE-MRI and DCECT was performed using an Olea sphere (Version 3.0, Olea Medical; La Ciotat, France). It was performed separately using four different decomposition methods: delay-sensitive and nonadaptive standard singular value decomposition (sSVD), delayinsensitive and non-adaptive circular singular value decomposition (cSVD), delay-insensitive and semi-adaptive oscillation index singular value decomposition (oSVD), and delay-insensitive and adaptive Bayesian decomposition. The singular value decomposition algorithms (SVD) have integrated an upstream threshold for the signal variance to reduce the effects of nonphysiological oscillations [21]. The Bayesian technique enables a rigorous probabilistic estimation of haemodynamic parameters based on Bayes' theorem [22]. In validated simulations, the Bayesian decomposition technique outperforms other techniques in terms of accuracy and robustness against noise [23].

Motion correction was applied automatically. To compare perfusion parameters derived from DCE-CT and DCE-MRI, the same ten time points were chosen to generate perfusion maps: $0,10,20,30,40,50,60,90,120$, and $180 \mathrm{~s}$ after contrast agent administration. To calculate the arterial input function (AIF), a region of interest (ROI) was placed manually in the radial artery. The venous output function (VOF) was determined in an ROI manually placed in the vein of the back of the hand. The baseline was defined as the time point at which increasing signal intensity was detectable. Colourcoded perfusion maps with 3-mm slice thickness were calculated for the following parameters: time to peak (TTP), which describes the time to the peak of contrast uptake in the selected ROI, relative blood volume ( $\mathrm{BBV})$, relative blood flow ( $\mathrm{rBF}$ ), and mean transit time (MTT). Joints with severe synovitis show increased perfusion parameters, indicated by red colour in the perfusion maps, while blue and black indicate regions with lower perfusion parameters (see Fig. 1).

\section{Image post-processing and regions of interest}

ROI analysis of the computed perfusion maps was performed using Osirix (Version 7.0, Pixmeo SARL; Bernex, Switzerland). Image post-processing was conducted in a coronal orientation. According to the established RAMRIS criteria [20], the ROIs were placed in MCP II, MCP III, MCP IV, MCP V, radioulnar, radiocarpal, and intercarpal joints. ROIs were placed in the region with the highest contrast uptake in inflamed joints. In noninflamed joints, ROIs were placed on the radial side of the joints and on the radial mediocarpal joints for the intercarpal region. All ROIs were circular, and an area of $10 \mathrm{~mm}^{2}$ was used for evaluation. The same ROI positioning was used for all four decomposition techniques and the two imaging modalities. The mean value of each measurement in each ROI was noted.

\section{Radiation exposure}

The radiation exposure (estimated effective dose) of DCE-CT examinations was calculated using the overall dose-length product (DLP) and a conversion coefficient of 0.0008 $\left[\mathrm{mSv} \times \mathrm{mGy}^{-1} \times \mathrm{cm}^{-1}\right]$. 
RS

T1w

CT

TTP

rBF

MTT MRI

CT
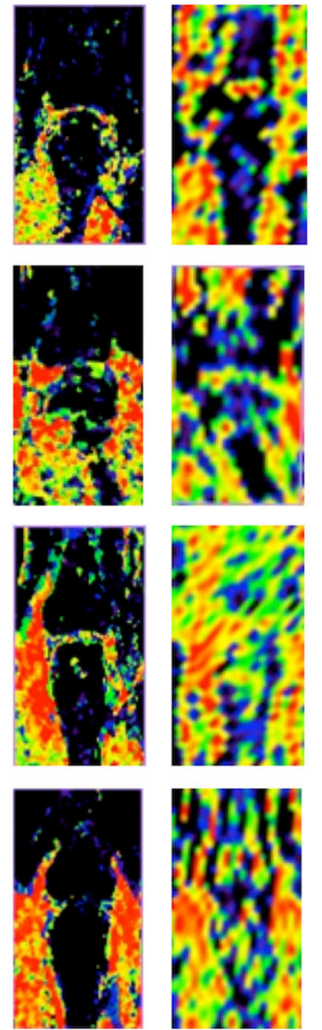
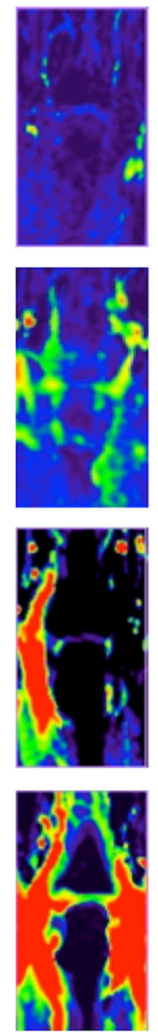
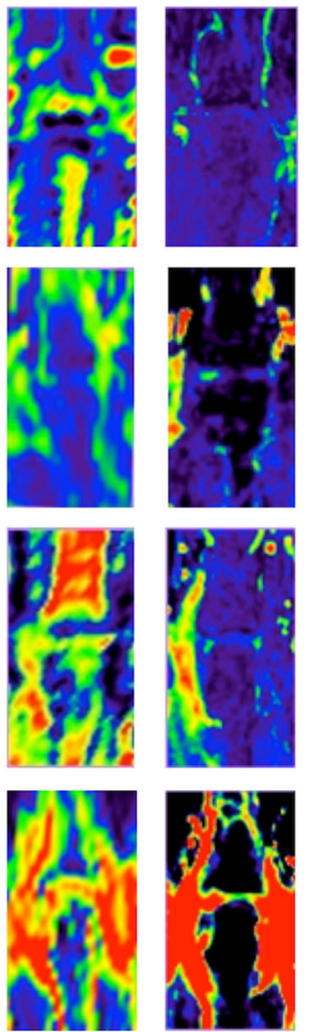

\section{CT}
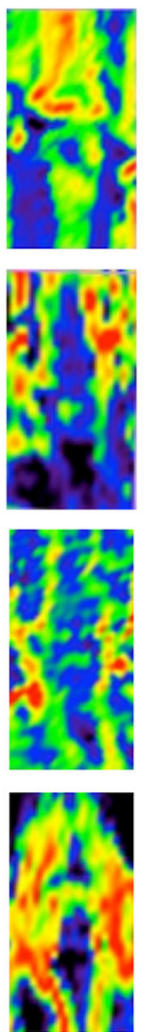
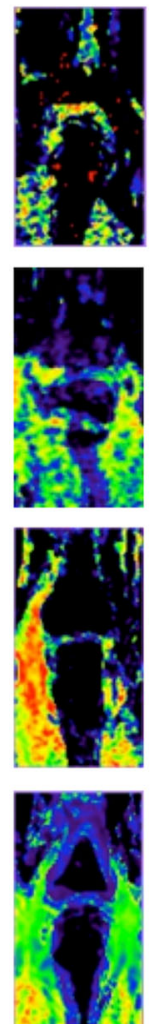

CT
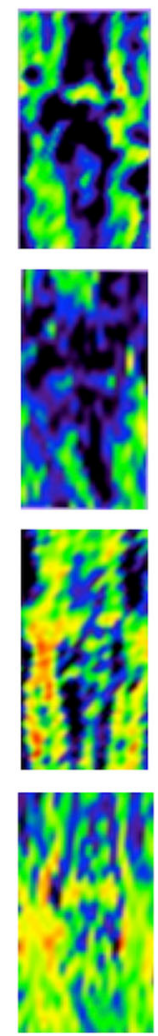

Fig. 1 Coronal perfusion maps of metacarpophalangeal joint III for different RAMRIS synovitis scores using standard singular value decomposition in four different patients. RS = RAMRIS synovitis score, $\mathrm{MRI}=$ magnetic resonance imaging, $\mathrm{CT}=$ computed tomography, $\mathrm{TTP}=$ time to peak, $\mathrm{rBV}=$ relative blood volume, $\mathrm{rBF}=$ relative blood flow, and

\section{Statistical analysis}

Statistical analysis was performed using R (RStudio, Version 1.1.463). The results of ROI analysis were collected and
MTT = mean transit time. The first column shows T1-weighted fat-saturated post-contrast image (T1w) and the second column shows contrastenhanced CT (CT) images. Perfusion parameters obtained with both modalities increase with RAMRIS scores. For RAMRIS scores 2 and 3, DCECT tends to underestimate the severity of synovitis compared to DCE-MRI summarized for the respective perfusion parameters. The mean and standard deviation were calculated for each perfusion parameter and decomposition technique separately. Negative values of ROI analysis due to artefacts were excluded from statistical

Table 2 Characteristics of the study population

\begin{tabular}{llll}
\hline Characteristics & Total & $R A$ & Non-RA \\
\hline No. of participants & 36 & 24 & 12 \\
Women & 26 & 17 & 9 \\
Men & 10 & 7 & 3 \\
Age (y) & $60.4 \pm 7.1(50-77)$ & $60.4 \pm 7.2(50-77)$ & $58.6 \pm 5.6(52-68)$ \\
Women & $59.2 \pm 6.2(50-74)$ & $58.8 \pm 6.6(50-74)$ & $60.0 \pm 5.7(52-68)$ \\
Men & $63.4 \pm 8.6(52-77)$ & $67.3 \pm 6.9(56-77)$ & $54.3 \pm 3.2(52-58)$ \\
Weight [kg] & $77.6 \pm 14.4$ & $79.7 \pm 15.3$ & $73.8 \pm 12.0$ \\
Symptom duration (y) & $4.2 \pm 4.9$ & $3.8 \pm 4.7$ & $5.0 \pm 5.4$ \\
CRP [mg/l] & $15.9 \pm 40.3$ & $21.9 \pm 47.0$ & $1.7 \pm 1.6$ \\
No. of ACPA-positive & 12 & 11 & 1 \\
No. of RF-positive & 8 & 8 & 0 \\
\hline
\end{tabular}

The values are given as follows: means \pm standard deviation and range in parentheses. $R A$ rheumatoid arthritis, $C R P C$-reactive protein, $A C P A$ anticitrullinated protein antibodies, $R F$ rheumatoid factors, ACPA-positive was defined as ACPA > $20 \mathrm{U} / \mathrm{ml}$ and RF-positive as RF > 14 U/ml) 
analysis. To identify significant differences between perfusion parameter pairs in DCE-CT and DCE-MRI, we performed a two-tailed paired $t$ test. The results of the paired t test were interpreted based on the effect size using Cohen's d with the following classification: no effect $<0.1$, small $0.2-0.4$, medium $0.5-0.7$, large $0.8-\geq 1.0$ [24]. Furthermore, means, standard deviations, and $95 \%$ confidence intervals were used for interpretation of differences. In a second step, an independent two-tailed $t$ test was used to investigate whether DCE-CT is able to differentiate between inflamed and noninflamed joints with a performance similar to that of DCE-MRI (according to scoring results). A $p$ value $<0.05$ was considered statistically significant.

For assessment of agreement between DCE-CT and DCEMRI, Bland-Altman plots of TTP, rBV, rBF, and MTT were created for inflamed joints separately for each of the four decomposition techniques.

\section{Results}

\section{Subjects and image reading}

The study included 37 patients (27 women; mean age 60.1, $50-77$ years) with a mean weight of $77.3 \mathrm{~kg}$ (SD 14.3), a mean C-reactive protein (CRP) of $18 \mathrm{mg} / \mathrm{dl}$ (SD 42.6), and a mean duration of symptoms of 3.9 years (SD 4.84). In one patient, DCE-MRI could not be performed due to technical problems during the examination and was therefore excluded from further statistical analysis. The study population and RAMRIS scores are presented in Table 2 and Fig. 2. ACPA was positive in 12 patients $(33.3 \%)$, while this was the case for RF in 8 patients $(22.25 \%)$. Fourteen patients were never treated, three were treated with NSAIDs, 6 received only corticosteroids, 7 received disease-modifying antirheumatic drugs (DMARDs),
Fig. 2 Flowchart of study inclusion and results of RAMRIS scoring for synovitis. $\mathrm{RA}=$ rheumatoid arthritis, $\mathrm{PsA}=$ psoriatic arthritis, $\mathrm{UA}=$ undifferentiated arthritis, $\mathrm{OA}=$ osteoarthritis, $\mathrm{CPPD}=$ calcium pyrophosphate deposition disease, $\mathrm{IC}=$ intercarpal joint, $\mathrm{RC}=$ radiocarpal joint, $\mathrm{RU}=$ radioulnar joint, and MCP2 $-5=$ metacarpophalangeal joints $2-5$

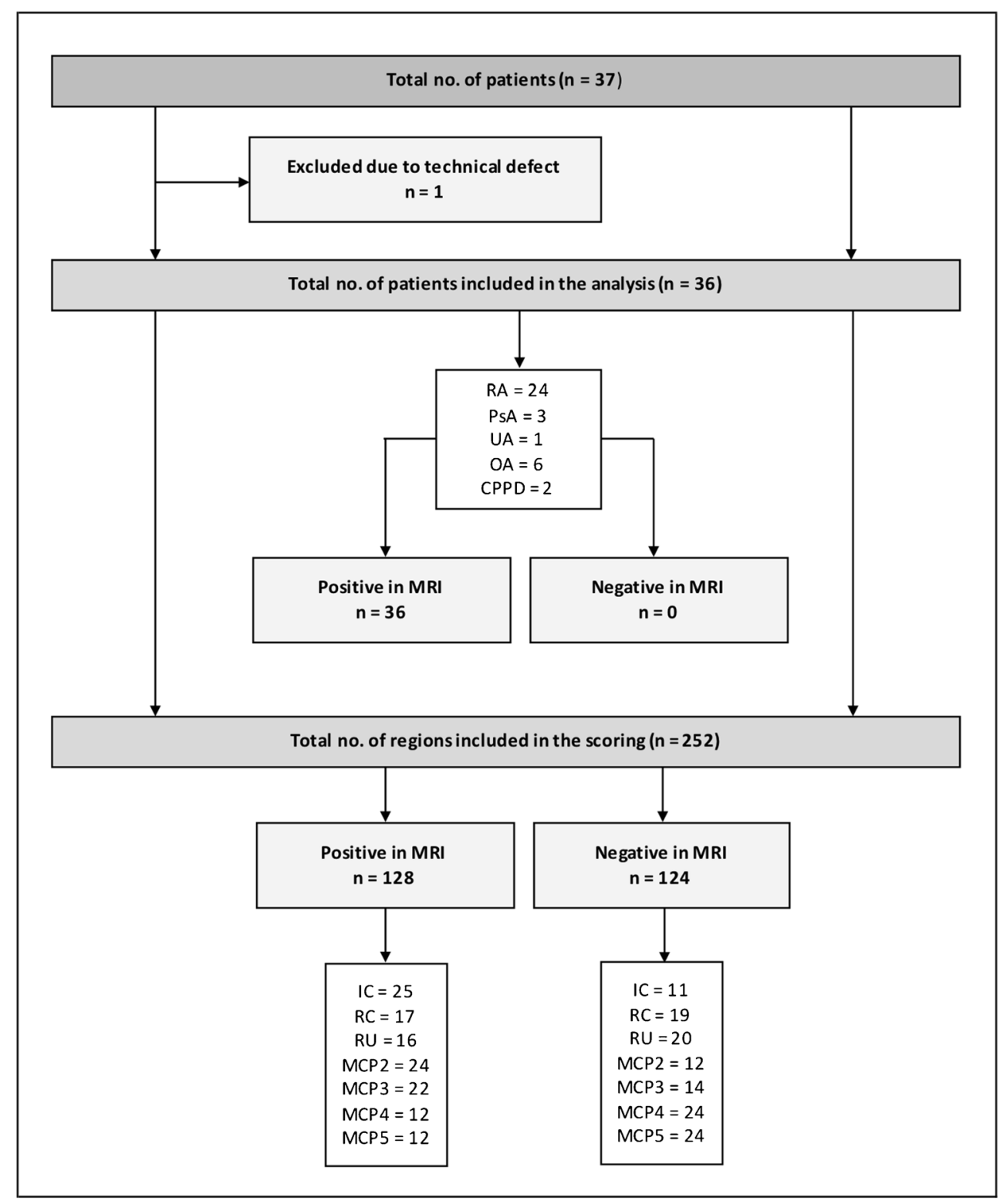


and 6 received biologicals. No patient received a corticosteroid injection prior to the examination.

Twenty-one patients underwent DCE-CT before DCEMRI. According to RAMRIS scores, 50.8\% (128/252) of the regions analysed were positive for synovitis (see Fig. 2).

\section{Image preprocessing}

In all patients, perfusion maps of TTP, rBV, $r B F$, and MTT were computed separately for preprocessing with SSVD, cSVD, oSVD, and the Bayesian algorithm. All data were accepted and successfully post-processed by the software. The post-processing and quantitative measurements took approximately 10 min per patient, modality, and reconstruction algorithm. These time, investments add up to approximately $48 \mathrm{~h}$ of continuous work for the whole study population. Examples of perfusion maps are shown in Fig. 1. In Fig. 3, boxplots of perfusion parameters of the different RAMRIS gradings are presented.

\section{Image post-processing and regions of interest}

Means, standard deviations, $\mathrm{t}$ test results, and effect sizes as well as differences in means and standard deviations of differences and 95\% confidence intervals of DCE-CT and DCE-MRI for TTP, rBV, rBF, and MTT using the different decomposition techniques are presented in Table 3. Overall, our results revealed no significant differences
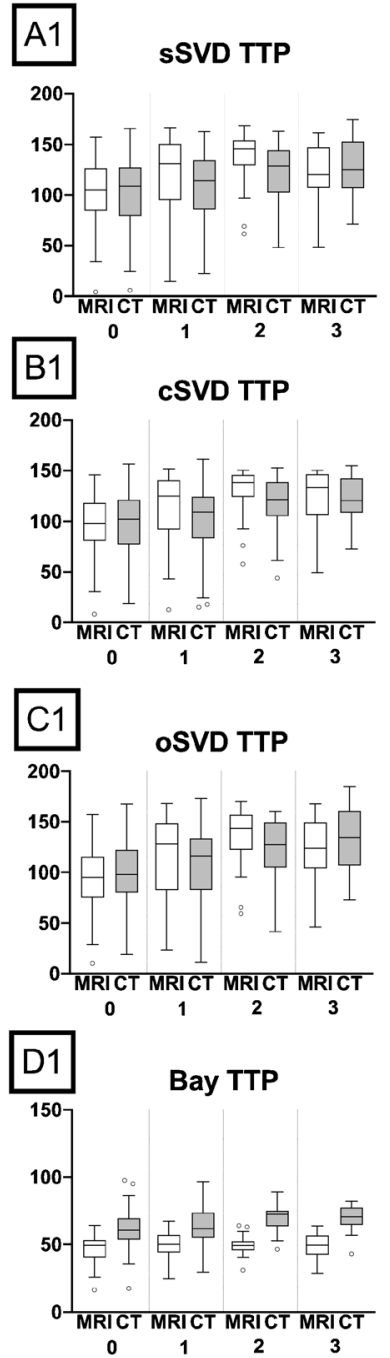
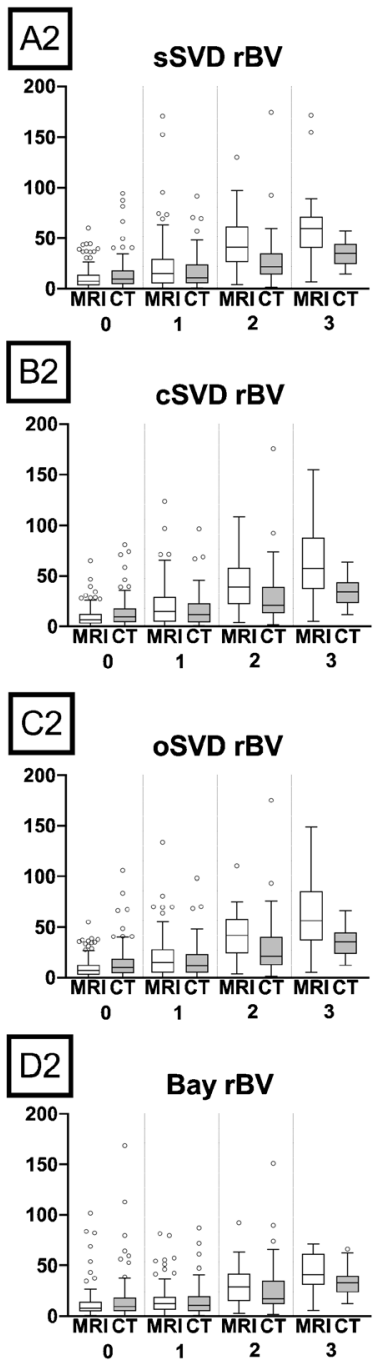

Fig. 3 Boxplots of perfusion parameters versus RAMRIS grading for the four decomposition techniques investigated. $\mathrm{SSVD}=$ standard singular value decomposition, $\mathrm{cSVD}=$ circular singular value decomposition, oSVD = oscillar singular value decomposition, Bay = Bayesian decomposition. $\mathrm{TTP}=$ time to peak, $\mathrm{rBV}=$ relative blood volume,
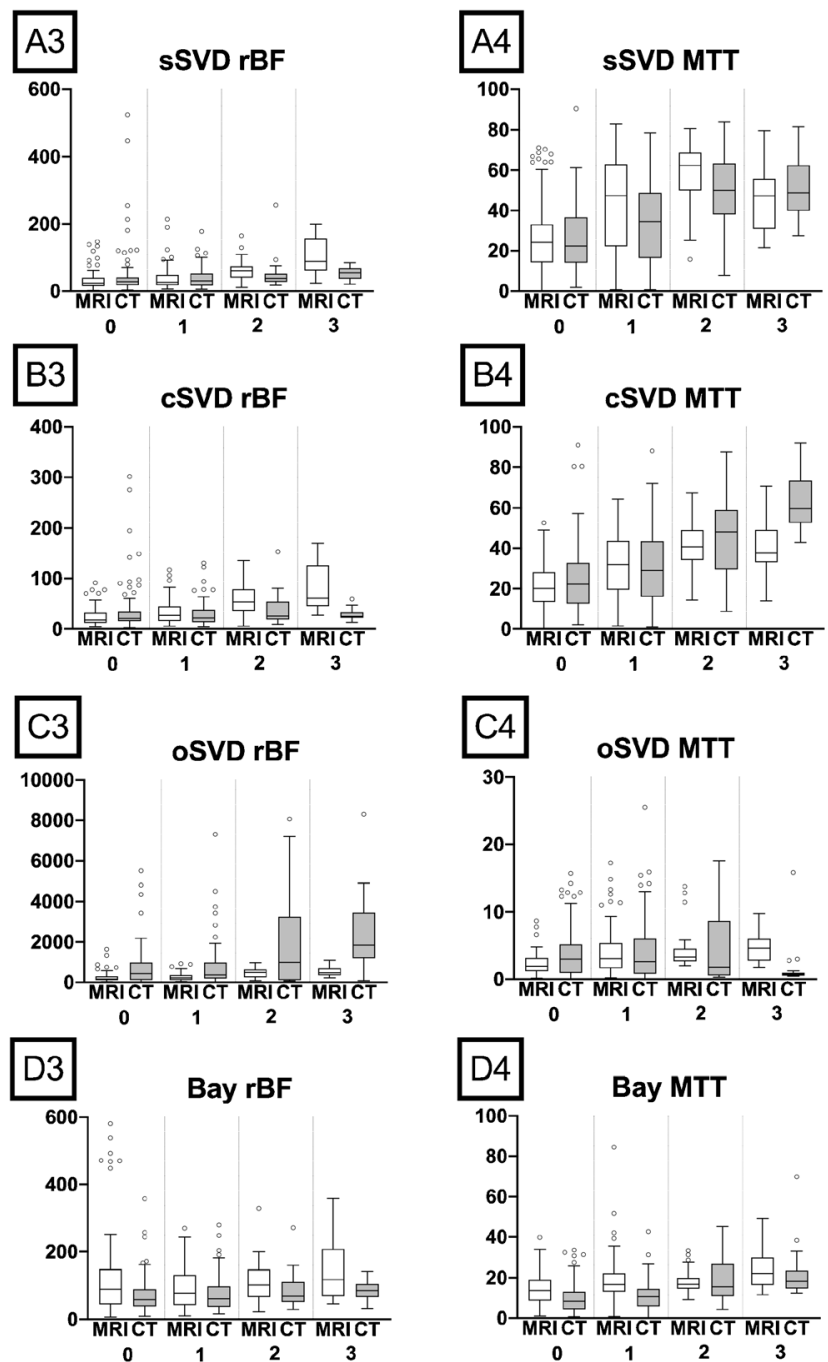

$\mathrm{rBF}=$ relative blood flow, and $\mathrm{MTT}=$ mean transit time. $\mathrm{Y}$ axis: perfusion parameter. $\mathrm{X}$ axis: RAMRIS synovitis score. Overall, perfusion parameters determined by DCE-CT and DCE-MRI increase with the RAMRIS synovitis score. However, for most parameters, DCE-CT seems to be less responsive than DCE-MRI 
Table 3 Results of the paired two-tailed t-test

\begin{tabular}{|c|c|c|c|c|c|c|c|c|c|c|}
\hline Decomp. & Param. & $\begin{array}{l}\text { MRI-derived } \\
\text { mean }\end{array}$ & $\begin{array}{l}\text { MRI-derived } \\
\text { SD }\end{array}$ & $\begin{array}{l}\text { CT-derived } \\
\text { mean }\end{array}$ & $\begin{array}{l}\text { CT-derived } \\
\text { SD }\end{array}$ & $\begin{array}{l}\text { Mean of } \\
\text { diff. }\end{array}$ & $\begin{array}{l}\text { SD of } \\
\text { diff. }\end{array}$ & $95 \%$ CI of diff. & $\mathrm{p}$ value & $\begin{array}{l}\text { Effect size } \\
\text { (Cohen's d) }\end{array}$ \\
\hline \multirow[t]{4}{*}{ sSVD } & ТTP & 116.2 & 32.7 & 110.1 & 32.4 & 6.1 & 40.6 & 0.9 to 11.2 & 0.019 & 0.2 \\
\hline & $\mathrm{rBV}$ & 24.5 & 29.1 & 19.1 & 20.1 & 5.4 & 25.3 & 2.2 to 8.5 & 0.001 & 0.2 \\
\hline & $\mathrm{rBF}$ & 43.7 & 38.9 & 44.5 & 53.4 & -0.7 & 60.0 & -8.2 to 6.8 & 0.845 & 0.01 \\
\hline & MTT & 37.5 & 22.0 & 34.0 & 19.9 & 3.5 & 23.6 & 0.5 to 6.5 & 0.020 & 0.2 \\
\hline \multirow[t]{4}{*}{ cSVD } & TTP & 110.3 & 30.4 & 103.6 & 32.4 & 6.7 & 38.4 & 1.9 to 11.5 & 0.007 & 0.2 \\
\hline & $\mathrm{rBV}$ & 22.4 & 26.0 & 18.8 & 19.9 & 3.6 & 23.3 & 0.7 to 6.5 & 0.015 & 0.2 \\
\hline & $\mathrm{rBF}$ & 36.4 & 30.9 & 32.6 & 34.8 & 3.8 & 43.9 & -1.7 to 9.2 & 0.176 & 0.09 \\
\hline & MTT & 28.8 & 15.0 & 32.3 & 20.5 & -3.5 & 19.2 & -5.9 to -1.1 & 0.004 & 0.2 \\
\hline \multirow[t]{4}{*}{ oSVD } & TTP & 109.8 & 36.1 & 108.6 & 33.8 & 1.2 & 42.5 & -4.0 to 6.5 & 0.644 & 0.03 \\
\hline & $\mathrm{rBV}$ & 22.7 & 25.6 & 19.6 & 20.9 & 3.1 & 23.9 & 0.1 to 6.1 & 0.044 & 0.1 \\
\hline & $\mathrm{rBF}$ & 307.3 & 248.2 & 1066.6 & 1480.8 & -759.2 & 1387.6 & -932.4 to -586.0 & $<0.001$ & 0.5 \\
\hline & MTT & 3.3 & 2.7 & 4.0 & 4.3 & -0.6 & 5.1 & -1.3 to 0.0005 & 0.050 & 0.1 \\
\hline \multirow[t]{4}{*}{ Bay } & TTP & 48.7 & 8.9 & 63.6 & 12.7 & -14.9 & 13.5 & -16.6 to -13.2 & $<0.001$ & 1.1 \\
\hline & $\mathrm{rBV}$ & 20.3 & 25.9 & 23.2 & 50.3 & -2.9 & 53.9 & -9.6 to 3.9 & 0.401 & 0.05 \\
\hline & $\mathrm{rBF}$ & 112.7 & 92.3 & 146.3 & 452.8 & -33.6 & 459.3 & -90.8 to 23.6 & 0.248 & 0.07 \\
\hline & MTT & 17.6 & 11.9 & 12.4 & 9.2 & 5.2 & 13.9 & 3.4 to 6.9 & $<0.001$ & 0.4 \\
\hline
\end{tabular}

Decomp. decomposition technique, Param. perfusion parameter, SD standard deviation, diff. difference, $95 \%$ CI 95\% confidence interval of the difference, $s S V D$ standard singular value decomposition, $c S V D$ circular singular value decomposition, $o S V D$ oscillar singular value decomposition, Bay Bayesian decomposition

between perfusion parameters determined with the two modalities, particularly for $\mathrm{rBF}$ in sSVD and TTP in oSVD. The observed differences were of small effect $(\mathrm{d}=0.01-0.4)$. The results indicate that DCE-CT is able to distinguish between inflamed and noninflamed joints (Table 4).

Table 4 Results of the independent t-test

\begin{tabular}{llllllll}
\hline Decomp. & Param. & +MRI mean \pm SD & -MRI mean \pm SD & MRI-derived p value & +CT mean \pm SD & -CT mean \pm SD & CT-derived p value \\
\hline sSVD & TTP & $126.2 \pm 32.0$ & $105.5 \pm 29.9$ & $<0.001$ & $116.5 \pm 31.7$ & $103.4 \pm 31.8$ & 0.001 \\
& rBV & $36.9 \pm 34.5$ & $10.9 \pm 11.6$ & $<0.001$ & $23.7 \pm 22.3$ & $14.2 \pm 16.1$ & $<0.001$ \\
& rBF & $55.4 \pm 45.0$ & $31.0 \pm 26.0$ & $<0.001$ & $44.6 \pm 33.3$ & $44.4 \pm 68.8$ & 0.982 \\
& MTT & $47.4 \pm 21.1$ & $26.6 \pm 17.4$ & $<0.001$ & $40.7 \pm 20.4$ & $26.7 \pm 16.5$ & $<0.001$ \\
cSVD & TTP & $120.9 \pm 29.4$ & $99.2 \pm 27.4$ & $<0.001$ & $109.5 \pm 31.6$ & $97.4 \pm 32.1$ & 0.003 \\
& rBV & $34.2 \pm 30.5$ & $9.8 \pm 10.2$ & $<0.001$ & $23.7 \pm 23.1$ & $13.7 \pm 14.2$ & $<0.001$ \\
& rBF & $48.5 \pm 36.1$ & $23.6 \pm 16.9$ & $<0.001$ & $31.2 \pm 24.1$ & $34.1 \pm 43.4$ & 0.522 \\
& MTT & $35.9 \pm 14.9$ & $21.3 \pm 11.1$ & $<0.001$ & $40.2 \pm 21.4$ & $24.0 \pm 15.8$ & $<0.001$ \\
0SVD & TTP & $123.0 \pm 35.6$ & $96.0 \pm 31.3$ & $<0.001$ & $116.5 \pm 34.2$ & $100.3 \pm 31.5$ & $<0.001$ \\
& rBV & $34.3 \pm 29.9$ & $10.3 \pm 10.5$ & $<0.001$ & $24.5 \pm 23.6$ & $14.4 \pm 16.2$ & $<0.001$ \\
& rBF & $372.9 \pm 254.1$ & $246.5 \pm 239.0$ & $<0.001$ & $1397.8 \pm 1809.4$ & $716.1 \pm 908.8$ & $<0.001$ \\
& MTT & $4.3 \pm 3.2$ & $2.3 \pm 1.4$ & $<0.001$ & $3.9 \pm 4.9$ & $4.0 \pm 3.7$ & 0.960 \\
Bay & TTP & $49.7 \pm 8.7$ & $47.7 \pm 9.0$ & 0.065 & $66.0 \pm 12.4$ & $61.1 \pm 12.6$ & 0.003 \\
& rBV & $27.7 \pm 30.9$ & $12.6 \pm 16.0$ & $<0.001$ & $22.5 \pm 21.5$ & $24.0 \pm 68.8$ & 0.818 \\
& rBF & $107.1 \pm 70.3$ & $119.2 \pm 110.0$ & 0.296 & $96.5 \pm 185.7$ & $198.7 \pm 616.7$ & 0.074 \\
& MTT & $20.5 \pm 14.1$ & $14.7 \pm 7.8$ & $<0.001$ & $14.9 \pm 10.2$ & $9.9 \pm 7.2$ & $<0.001$ \\
\hline
\end{tabular}

Decomp. decomposition technique, Param. perfusion parameter, $+M R /+C T$ results for inflamed joints in $\mathrm{MRI}$ und $\mathrm{CT},-M R I /-C T$ results for noninflamed joints, $S D$ standard deviation, $s S V D$ standard singular value decomposition, $c S V D$ circular singular value decomposition, $o S V D$ oscillar singular value decomposition, Bay Bayesian decomposition 
Figure 4 presents Bland-Altman plots for TTP, rBV, rBF, and MTT in regions with synovitis for each of the four decomposition techniques, namely, sSVD, oSVD, cSVD, and Bayesian technique. The plots show that joints with severe inflammation tended to be underestimated in DCE-CT.

\section{Radiation exposure}

The total DLP was $48 \mathrm{mGy}^{*} \mathrm{~cm}$, resulting in an estimated effective dose of $0.038 \mathrm{mSv}$.

\section{Discussion}

This is the first study investigating the innovative technique of low-dose DCE-CT for the assessment of synovitis of the hand in patients with active arthritis using four different decomposition techniques. We show that DCE-CT is able to differentiate between inflamed and noninflamed joints and between the different grades of the semi-quantitative RAMRIS synovitis scores with no major differences between the four

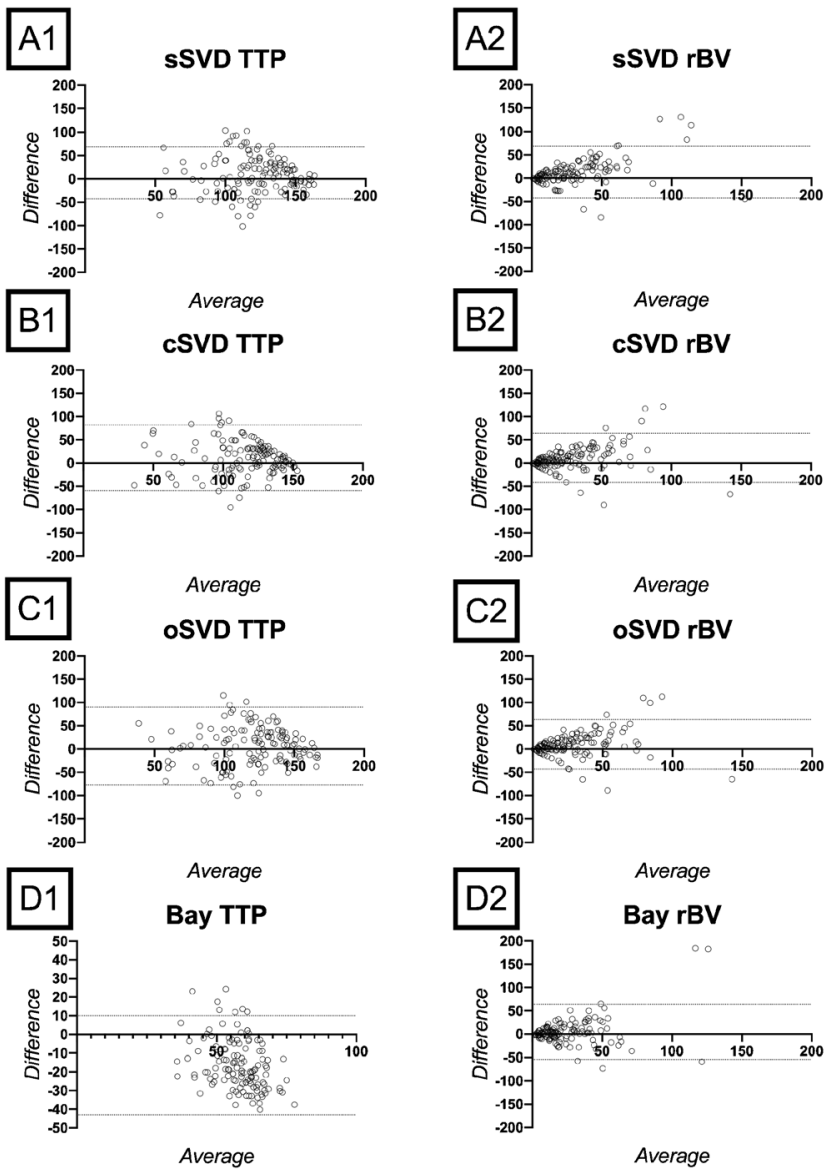

Fig 4 Bland-Altman plots. sSVD = standard singular value decomposition, $\mathrm{cSVD}=$ circular singular value decomposition, $\mathrm{OSVD}=$ oscillar singular value decomposition, Bay $=$ Bayesian decomposition. $\mathrm{TTP}=$ time to peak, $\mathrm{rBV}=$ relative blood volume, $\mathrm{rBF}=$ relative blood decomposition techniques studied. While DCE-CT tends to underestimate severe inflammation, it provides similar perfusion parameters as DCE-MRI and is able to identify joints with synovitis. In this regard, rBF shows the best agreement between the two modalities. Differences in perfusion parameters identified between the two modalities have small effects.

DCE-CT is more sensitive than MRI or traditional radiography in detecting soft tissue calcification and smaller erosions. Thus, $\mathrm{CT}$ allows a broader differential diagnosis. Due to the upcoming ultra-low-dose protocols [25], CT has the potential to be applied in routine care. DCE-CT also allows automated image post-processing and reading. Furthermore, it also offers a gadolinium-free alternative, as MRI contrast media can result in gadolinium deposition with as yet unclear effects [26, 27]. Additionally, CT is faster and more readily available. In our previous study, we showed that patients prefer CT than MRI and feel more comfortable in a CT scanner [13]. For comparability with MRI, CT examinations in our study were performed in the superman position as well. In the clinical setting, CT examinations can be performed with the patient sitting comfortably next to the gantry.

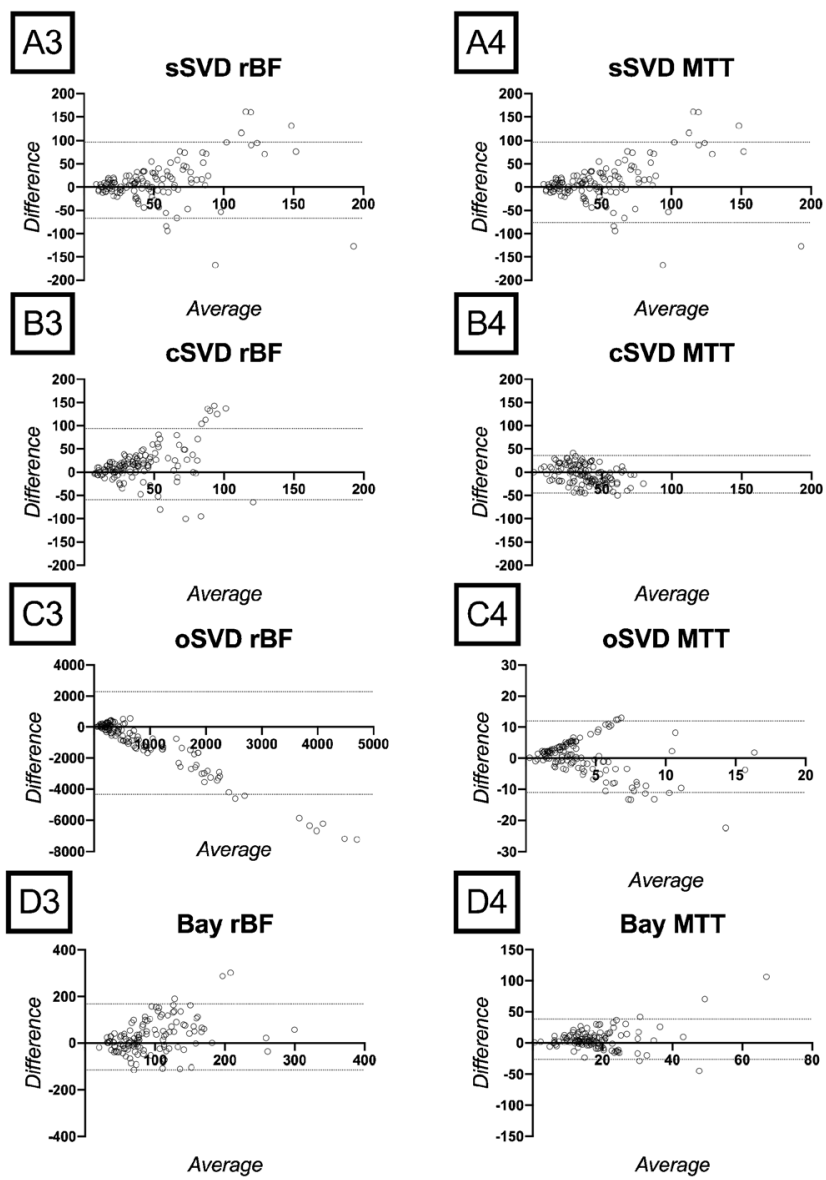

flow, and MTT $=$ mean transit time. $\mathrm{Y}$ axis: difference between DCEMRI and DCE-CT. X axis: average DCE-MRI and DCE-CT. There is good overall agreement between the two modalities, but DCE-CT tends to underestimate disease activity in joints with severe inflammation 
Sonography has several advantages in the detection of synovial inflammation using power-Doppler with a high diagnostic ability in depicting erosions, synovial hypertrophy, and hyperaemia [28]. However, its diagnostic performance varies with the examiner's experience [11], and the quantification of inflammation and structural lesions for follow-up is limited [29]. For this reason, a comparison of sonography with DCE-CT and DCE-MRI is unfeasible. Modern CT techniques allow the depiction of bone marrow oedema [30]. DCE and dual-energy CT might help to detect osteitis, and the combined use of both techniques may provide further benefits.

Our study was specifically planned to compare DCE-CT and DCE-MRI. We used adapted doses of contrast agent in DCE-CT and DCE-MRI to create comparable conditions. The same software was used to generate perfusion maps in DCE$\mathrm{CT}$ and DCE-MRI and for quantitative ROI analysis. However, our study has some limitations. The different inherent properties of the imaging modalities and contrast agents must be taken into account [31]. We investigated only 36 patients, adhering to our estimate of the necessary sample size. We are aware that, despite showing the absence of significant differences in perfusion parameter means between $\mathrm{CT}$ and MRI, our study is not designed to establish equivalence or non-inferiority. Gadolinium retention in patients who underwent MRI first might have influenced the CT enhancement. Using a low-dose CT protocol can both limit the ability to detect subtle bone changes and lead to measurement artefacts that can be minimized by applying higher radiation to improve the contrast-to-noise ratio. According to the new EULAR recommendation, MRI is no longer used for the early diagnosis of RA because of its low specificity [32]. Further studies investigating DCE-CT using an optimized image reconstruction protocol (e.g. artificial intelligence) in larger numbers of patients are needed. In addition, a comparison with $3 \mathrm{~T}$ scanners would be of particular interest.

DCE-CT has the potential to assess synovitis. Using four decomposition techniques, we showed that there is overall good agreement between DCE-CT and DCE-MRI, while DCE-CT tends to underestimate some perfusion parameters. In patients with a contraindication to MRI or when MRI is not available, DCE-CT is a suitable alternative to detect and assess arthritis.

In conclusion, low-dose DCE-CT can differentiate inflamed and noninflamed joints in patients with suspected RA. Additionally, DCE-CT produces perfusion parameters comparable with DCE-MRI.

Acknowledgements The authors thank Mrs. Bettina Herwig for language editing. Ralph Schilling kindly provided statistical advice for this manuscript.

Funding Information Open Access funding provided by Projekt DEAL. The study was supported by Canon Medical Systems, who provided software for the reconstruction of perfusion maps.

\section{Compliance with ethical standards}

Conflict of interest The authors declare that they have no conflicts of interest. The study was supported by Canon Medical Systems. However, the company did not influence or authorize the study results.

Ethical approval All procedures performed in studies involving human participants were in accordance with the ethical standards of the institutional and/or national research committee and with the 1964 Helsinki declaration and its later amendments or comparable ethical standards.

Informed consent Informed consent was obtained from all individual participants included in the study.

Open Access This article is licensed under a Creative Commons Attribution 4.0 International License, which permits use, sharing, adaptation, distribution and reproduction in any medium or format, as long as you give appropriate credit to the original author(s) and the source, provide a link to the Creative Commons licence, and indicate if changes were made. The images or other third party material in this article are included in the article's Creative Commons licence, unless indicated otherwise in a credit line to the material. If material is not included in the article's Creative Commons licence and your intended use is not permitted by statutory regulation or exceeds the permitted use, you will need to obtain permission directly from the copyright holder. To view a copy of this licence, visit http://creativecommons.org/licenses/by/4.0/.

\section{References}

1. Scott DL, Wolfe F, Huizinga TW. Rheumatoid arthritis. Lancet. 2010;376(9746):1094-108.

2. Kay J, Upchurch KS. ACR/EULAR 2010 rheumatoid arthritis classification criteria. Rheumatology (Oxford). 2012;51(Suppl 6):vi59.

3. Axelsen MB, Poggenborg RP, Stoltenberg M, Kubassova O, Boesen M, Horslev-Petersen K, et al. Reliability and responsiveness of dynamic contrast-enhanced magnetic resonance imaging in rheumatoid arthritis. Scand J Rheumatol. 2013;42(2):115-22.

4. Wojciechowski W, Tabor Z, Urbanik A. Assessing synovitis based on dynamic gadolinium-enhanced MRI and EULAR-OMERACT scores of the wrist in patients with rheumatoid arthritis. Clin Exp Rheumatol. 2013;31(6):850-6.

5. Deniffel D, Boutelier T, Labani A, Ohana M, Pfeiffer D, Roy C. Computed tomography perfusion measurements in renal lesions obtained by Bayesian estimation, advanced singular-value decomposition deconvolution, maximum slope, and Patlak models: intermodel agreement and diagnostic accuracy of tumor classification. Investig Radiol. 2018;53(8):477-85.

6. Axelsen MB, Ejbjerg BJ, Hetland ML, Skjodt H, Majgaard O, Lauridsen UB, et al. Differentiation between early rheumatoid arthritis patients and healthy persons by conventional and dynamic contrast-enhanced magnetic resonance imaging. Scand J Rheumatol. 2014;43(2):109-18.

7. Navalho M, Resende C, Rodrigues AM, Gaspar A, Fonseca JE, Canhao H, et al. Dynamic contrast-enhanced 3-T magnetic resonance imaging: a method for quantifying disease activity in early polyarthritis. Skelet Radiol. 2012;41(1):51-9.

8. van de Sande MG, van der Leij C, Lavini C, Wijbrandts CA, Maas M, Tak PP. Characteristics of synovial inflammation in early arthritis analysed by pixel-by-pixel time-intensity curve shape analysis. Rheumatology (Oxford). 2012;51(7):1240-5. 
9. Waterton JC, Ho M, Nordenmark LH, Jenkins M, DiCarlo J, Guillard G, et al. Repeatability and response to therapy of dynamic contrast-enhanced magnetic resonance imaging biomarkers in rheumatoid arthritis in a large multicentre trial setting. Eur Radiol. 2017;27(9):3662-8.

10. Kawashiri SY, Suzuki T, Nakashima Y, Horai Y, Okada A, Nishino A, et al. Synovial inflammation assessed by ultrasonography correlates with MRI-proven osteitis in patients with rheumatoid arthritis. Rheumatology (Oxford). 2014;53(8):1452-6.

11. Hoving JL, Buchbinder R, Hall S, Lawler G, Coombs P, McNealy $\mathrm{S}$, et al. A comparison of magnetic resonance imaging, sonography, and radiography of the hand in patients with early rheumatoid arthritis. J Rheumatol. 2004;31(4):663-75.

12. Fukuda T, Umezawa Y, Tojo S, Yonenaga T, Asahina A, Nakagawa $\mathrm{H}$, et al. Initial experience of using dual-energy $\mathrm{CT}$ with an iodine overlay image for hand psoriatic arthritis: comparison study with contrast-enhanced MR imaging. Radiology. 2017; 161671 .

13. Diekhoff T, Ulas ST, Poddubnyy D, Schneider U, Hermann S, Biesen R, et al. Ultra-low-dose CT detects synovitis in patients with suspected rheumatoid arthritis. Ann Rheum Dis. 2019;78(1):31-5.

14. Dohn UM, Ejbjerg BJ, Hasselquist M, Narvestad E, Moller J, Thomsen HS, et al. Detection of bone erosions in rheumatoid arthritis wrist joints with magnetic resonance imaging, computed tomography and radiography. Arthritis Res Ther. 2008;10(1):R25.

15. Topfer D, Finzel S, Museyko O, Schett G, Engelke K. Segmentation and quantification of bone erosions in highresolution peripheral quantitative computed tomography datasets of the metacarpophalangeal joints of patients with rheumatoid arthritis. Rheumatology (Oxford). 2014;53(1):65-71.

16. de Bucourt M, Scheurig-Munkler C, Feist E, Juran R, Diekhoff T, Rogalla $\mathrm{P}$, et al. Cyst-like lesions in finger joints detected by conventional radiography: comparison with 320-row multidetector computed tomography. Arthritis Rheum. 2012;64(4):1283-90.

17. Vardhanabhuti V, Riordan RD, Mitchell GR, Hyde C, Roobottom CA. Image comparative assessment using iterative reconstructions: clinical comparison of low-dose abdominal/pelvic computed tomography between adaptive statistical, model-based iterative reconstructions and traditional filtered back projection in 65 patients. Investig Radiol. 2014;49(4):209-16.

18. Gervaise A, Osemont B, Lecocq S, Noel A, Micard E, Felblinger J, et al. CT image quality improvement using adaptive iterative dose reduction with wide-volume acquisition on 320-detector CT. Eur Radiol. 2012;22(2):295-301.

19. Aletaha D, Neogi T, Silman AJ, Funovits J, Felson DT, Bingham CO 3rd, et al. 2010 rheumatoid arthritis classification criteria: an American College of Rheumatology/European league against rheumatism collaborative initiative. Ann Rheum Dis. 2010;69(9):1580 8.

20. Ostergaard M, Edmonds J, McQueen F, Peterfy C, Lassere M, Ejbjerg B, et al. An introduction to the EULAR-OMERACT rheumatoid arthritis MRI reference image atlas. Ann Rheum Dis. 2005;64(Suppl 1):i3-7.

21. Kudo K, Boutelier T, Pautot F, Honjo K, Hu JQ, Wang HB, et al. Bayesian analysis of perfusion-weighted imaging to predict infarct volume: comparison with singular value decomposition. Magn Reson Med Sci. 2014;13(1):45-50.

22. Sasaki M, Kudo K, Boutelier T, Pautot F, Christensen S, Uwano I, et al. Assessment of the accuracy of a Bayesian estimation algorithm for perfusion CT by using a digital phantom. Neuroradiology. 2013;55(10):1197-203.

23. Kudo K, Christensen S, Sasaki M, Ostergaard L, Shirato H, Ogasawara K, et al. Accuracy and reliability assessment of CT and MR perfusion analysis software using a digital phantom. Radiology. 2013;267(1):201-11.

24. Cohen J. Statistical power analysis for the behaviors science.(2nd). New Jersey: Laurence Erlbaum Associates, Publishers, Hillsdale; 1988.

25. Lin S, Lin M, Lau KK. Image quality comparison between modelbased iterative reconstruction and adaptive statistical iterative reconstruction chest computed tomography in cystic fibrosis patients. J Med Imaging Radiat Oncol. 2019;63(5):602-9.

26. Ramalho J, Semelka RC, AlObaidy M, Ramalho M, Nunes RH, Castillo M. Signal intensity change on unenhanced T1-weighted images in dentate nucleus following gadobenate dimeglumine in patients with and without previous multiple administrations of gadodiamide. Eur Radiol. 2016;26(11):4080-8.

27. Bae S, Lee HJ, Han K, Park YW, Choi YS, Ahn SS, et al. Gadolinium deposition in the brain: association with various GBCAs using a generalized additive model. Eur Radiol. 2017;27(8):3353-61.

28. Moller I, Janta I, Backhaus M, Ohrndorf S, Bong DA, Martinoli C, et al. The 2017 EULAR standardised procedures for ultrasound imaging in rheumatology. Ann Rheum Dis. 2017;76(12):1974-9.

29. Caporali R, Smolen JS. Back to the future: forget ultrasound and focus on clinical assessment in rheumatoid arthritis management. Ann Rheum Dis. 2018;77(1):18-20.

30. Jans L, De Kock I, Herregods N, Verstraete K, Van den Bosch F, Carron P, et al. Dual-energy CT: a new imaging modality for bone marrow oedema in rheumatoid arthritis. Ann Rheum Dis. 2018;77(6):958-60.

31. Ng CS, Waterton JC, Kundra V, Brammer D, Ravoori M, Han L, et al. Reproducibility and comparison of DCE-MRI and DCE-CT perfusion parameters in a rat tumor model. Technol Cancer Res Treat. 2012;11(3):279-88.

32. Combe B, Landewe R, Daien CI, Hua C, Aletaha D, Alvaro-Gracia JM, et al. 2016 update of the EULAR recommendations for the management of early arthritis. Ann Rheum Dis. 2017;76(6):94859.

Publisher's note Springer Nature remains neutral with regard to jurisdictional claims in published maps and institutional affiliations. 